\title{
Extraction method of weak underwater acoustic signal based on the combination of wavelet transform and empirical mode decomposition
}

\author{
Junbing Shi ${ }^{*}$, Yingmin Wang, Xiaoyong Zhang, and Libo Yang \\ Department of Intelligence and Automation, Taiyuan University, Taiyuan 030032, China
}

Received: 25 December 2020 / Accepted: 3 March 2021

\begin{abstract}
When studying underwater acoustic exploration, tracking and positioning, the target signals collected by hydrophones are often submerged in strong intermittent noise and environmental noise. In this paper, an algorithm that combines empirical mode decomposition and wavelet transform is proposed to achieve the efficient extraction of target signals in the environment with strong noise. First the calibration of baseline drift is performed on the algorithm, and then it is decomposed into different intrinsic mode functions via empirical mode. The wavelet threshold processing is conducted according to the correlation coefficient of each mode component and the original signal, and finally the signals are reconstructed. The simulation and experiment results show that compared with the conventional empirical mode decomposition method and wavelet threshold method, when the signal-to-noise ratio is low and there exist high-frequency intermittent jamming and baseline drift, the combined algorithm can better extract the target signal, laying the foundation for direction-of-arrival estimation and target positioning in the next step.
\end{abstract}

Keywords: Signal-to-noise ratio / empirical mode decomposition / wavelet transform / intrinsic mode component

\section{Introduction}

Since the 21st century, ocean has played an increasing important role in the development of mankind, and industries such as marine resource acquisition and marine transportation have developed rapidly. Among ocean technologies, passive sonar detection technology is a key technology for humans to better exploit and utilize ocean, which has become an important research field [1-3]. As for sonar technology, underwater acoustic signal receiving and processing technology is an important content [4]. The acquisition and processing of acoustic signal determines the development of sonar technology, thus affecting the exploitation of marine resources. At present, vector hydrophone is a research focus, whose basic concept is to confirm the azimuth information of underwater target by collecting vector information such as acoustic pressure information and vibration speed of the sound field. The vector hydrophones that are mostly studied and develop rapidly include co-vibration vector hydrophone, optical fiber vector hydrophone and MEMS vector hydrophone

* Corresponding author: bestxj1@qq.com
[5-7], whose sensors with higher sensitivity and smaller structure are suitable for multi-platform operation with stable performance [8]. Due to the complex underwater environment, the collected underwater acoustic information often contains a lot of noise, and the signal is sometimes completely submerged in the noise, so it is critical to reduce the noise of the signal. The signal characteristics are not obvious in the strong noise background with low signal-to-noise ratio [9], so the Fourier transform signal processing method used has a poor processing effect in the actual strong noise environment.

\section{Noise reduction principle and combined algorithm}

The collected signal usually contains a lot of noise, so noise reduction is important for the extraction of underwater acoustic signals. In practices, noise reduction is critical for target detection and orientation. In order to improve the accuracy of target detection and orientation, it is necessary to enhance the noise reduction ability [10]. In the noise reduction process, the signal-to-noise ratio, root mean square error (RMSE) and signal-to-noise ratio gain $(\mathrm{G})$ are 
mainly used to measure the performance of the algorithm. The expressions are shown in equations (1)-(3).

$$
\begin{gathered}
\mathrm{SNR}_{\text {out }}=10 * \log \frac{S}{N} \\
\mathrm{RMSE}=\sqrt{\frac{\sum_{n=1}^{L}\left(X_{n}-\widehat{X}_{n}\right)^{2}}{L}} \\
G=\mathrm{SNR}_{\text {out }}-\mathrm{SNR}_{\text {in }}
\end{gathered}
$$

where $\mathrm{SNR}_{\text {out }}$ is the output signal-to-noise ratio, $S$ is the signal power, and $N$ is the noise power. The actual calculated value is $X_{n}$, the true value is $\widehat{X}_{n}$, and the number of observations is $L$. The larger the output signal-to-noise ratio, the stronger the algorithm's anti-interference ability, the better the target resolution ability, and the better the algorithm's noise reduction performance. The smaller the root mean square error, the more stable the algorithm. The higher the signal-to-noise ratio gain, the better the performance of the processing algorithm.

\subsection{EMD method}

The Empirical Mode Decomposition (EMD) method is a signal decomposition method proposed by Huang, which has an excellent effect of processing non-stationary signals [11]. During EMD processing, the signal is first decomposed into a series of intrinsic mode components and a margin [12], and then the effective mode is reconstructed to achieve signal denoising optimization. It is mainly used to identify the natural mode of vibration based on the time scale features contained in the data, so as to realize the decomposition of the data. The intrinsic mode function must meet two conditions: (a) within the signal range, the number of extreme point and zero-crossing points are equal or differ by one at most; (b) at any point in time, the mean value between the upper and the lower envelope determined by the maximum value and minimum value respectively is zero. The expression is shown in equation (4).

$$
x(t)=\sum_{i=1}^{n} i m f_{i}(t)+r(t)
$$

where $i m f_{i}(t)$ represents the $i$ th intrinsic mode component, $r(t)$ represents the trend component, and $n$ indicates the number of layers of adaptive decomposition.

\subsection{Wavelet threshold processing}

The wavelet analysis is to perform the Fourier transform based on the original collected signal, and then realize the multi-scale detailed analysis of the signal via processing such as expansion and translation [13,14]. Wavelet denoising is performed through shortwave to remove noise. Wavelet transform can remove signal correlation, and noise has a tendency to whiten after wavelet transform, so it is

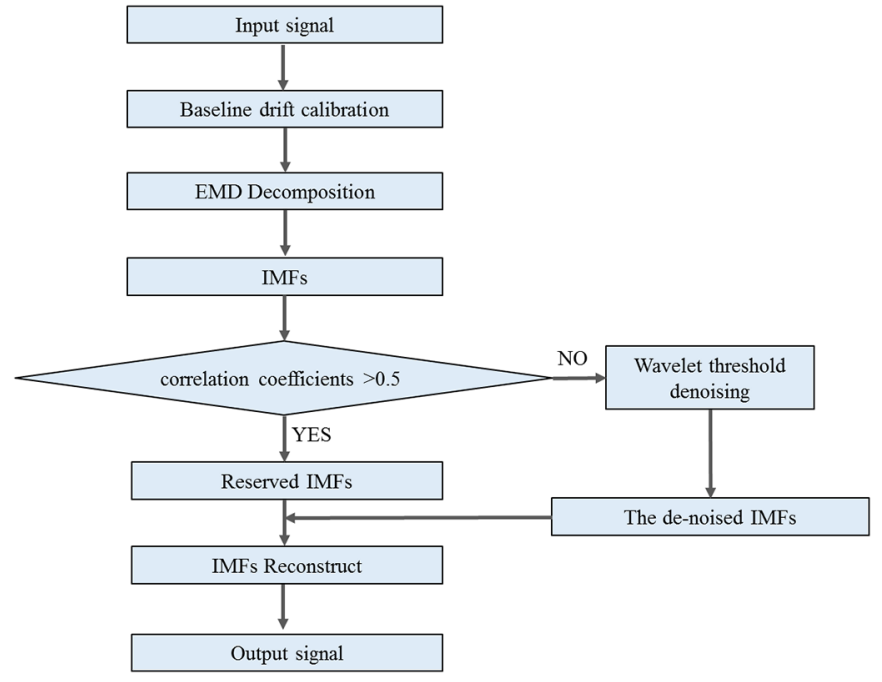

Fig. 1. Algorithm flow diagram.

more conducive to denoising than time domain; The core of wavelet transform is primary function, and the selection of primary function determines the result of signal analysis. Hence, the selection of primary function is critical. In practice, the appropriate wavelet primary function is selected according to characteristics of signal and denoising requirements. In this paper, the "db4" wavelet primary function is selected, and the threshold denoising is to process the coefficients of each layer after wavelet decomposition that are greater and less than a certain threshold. Based on experience, the soft threshold denoising rule is selected for signal processing, where the improved soft threshold function and threshold expression are as follows

$$
\widehat{u}_{j}=\left\{\begin{array}{c}
\operatorname{sign}\left(u_{j}\right)\left(\left|u_{j}\right|-\frac{\mu}{\exp \left(\frac{\left|u_{j}\right|-\mu}{N}\right)}\right), \quad\left|u_{j}\right| \geq \mu \\
0, \quad\left|u_{j}\right|<\mu \\
\mu=\frac{\sigma \sqrt{2 \log N}}{\log (j+1)}
\end{array}\right.
$$

where $\widehat{u}_{j}$ is the threshold function, $\mu$ is the threshold, $N$ is the data length, and $j$ is the wavelet processing decomposition coefficient.

\subsection{Extraction method of weak underwater acoustic signal based on the combination of wavelet transform and EMD}

According to the characteristics of EMD decomposition method and wavelet transform, the flow diagram of the proposed combined algorithm is shown in Figure 1. The basic processing flow of the algorithm is: 

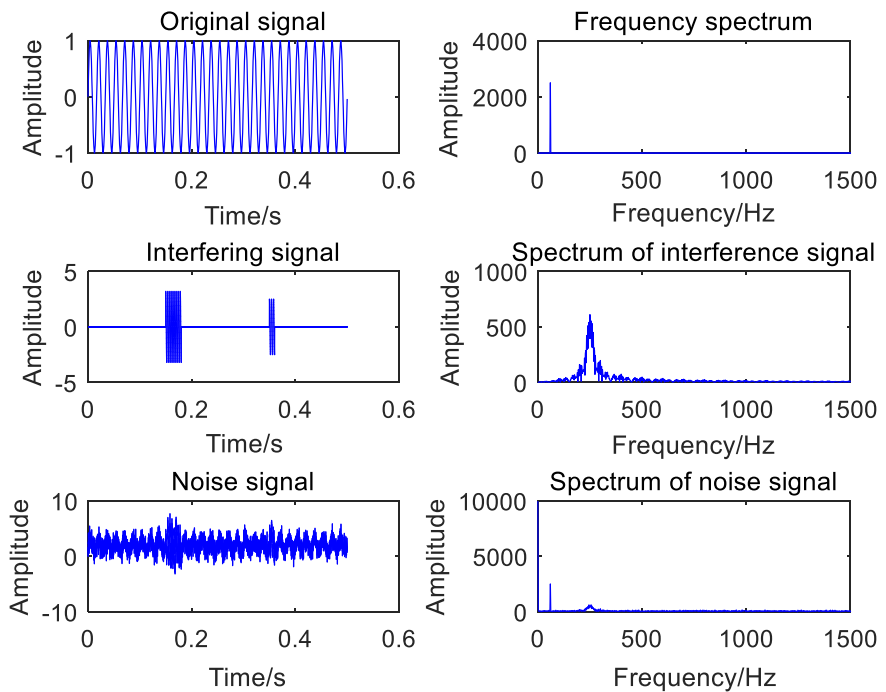

Fig. 2. Time domain and frequency domain waveforms of the signal.

- The collected original signal is taken as the input signal to perform calibration of baseline drift to remove the DC offset component in the signal;

- EMD is adopted to decompose the preprocessed signal to calculate multiple intrinsic mode components;

- The useful mode component is judged by the correlation coefficient between the intrinsic mode component and the original signal. The larger the correlation coefficient, the higher the effective component of the signal. In this paper, 0.5 is selected as the judgment threshold of correlation coefficient;

- Under the conditions of pre-selected wavelet primary function and threshold rules, perform wavelet de-noising on the noise component to obtain the signal after the second processing;

- The second output signal is reconstructed again, and finally the best estimate of the target signal is extracted.

This algorithm is based on EMD decomposition transform and wavelet threshold processing. By combining the advantages of the two, the signal is nested multiple times to obtain the best estimate of the target signal.

\section{Simulation and experiment result analysis}

This experiment uses MATLAB and its signal processing toolbox function to verify and simulate the algorithm proposed in this paper [15]. Firstly, the impact of highfrequency intermittent signals on the extraction of target signals under different signal-to-noise ratios are taken into consideration. Assume that the signal-to-noise ratio is $0 \mathrm{db}$, the target signal is a $60 \mathrm{~Hz}$ sinusoidal signal, and the high frequency intermittent interference is $250 \mathrm{~Hz}$ sinusoidal signal, which are superimposed to the received signal by hydrophones at different times. The total length of the data is 0.5 seconds, and the sampling rate is $10 \mathrm{kHz}$. The time domain and frequency domain results of the signal are shown in Figure 2. The left side is the time domain signal,
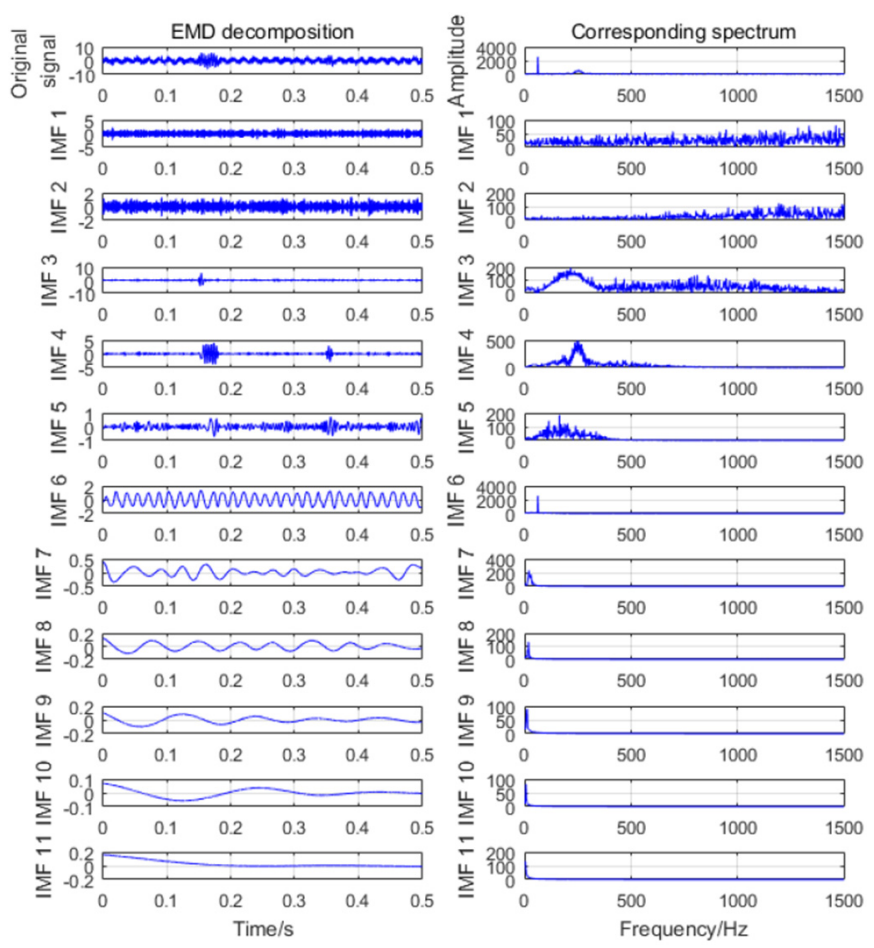

Fig. 3. EMD signal decomposition time domain and frequency domain diagram.

the horizontal ordinate is the sampling time, and the vertical ordinate is the voltage magnitude. The right side is the signal frequency domain diagram, the horizontal ordinate is the frequency, and the vertical ordinate is the frequency spectrum of the signal, that is, the magnitude after Fourier transform. The first line is the original target signal, the second line is the waveform diagram of the highfrequency intermittent signal, and the third line is the signal diagram after Gaussian white noise and the intermittent signal are superposed.

Secondly, the calibration of baseline drift is performed on the signal, the signal is calibrated to the zero position, and the DC offset component is removed. Then the preprocessed signal containing noise and interference is decomposed by EMD, and it is adaptively decomposed into 11 layers. The decomposition structure is shown in Figure 3. Each layer represents a different frequency band signal. It can be seen that the first two orders of intrinsic mode components contain Gaussian noise, and the third and fourth order modes are mainly high-frequency intermittent signals. The other high-order signals are all extremely low frequency noise signals. By calculating the correlation coefficient between each IMF component and the original signal, the information component contained in each mode can be determined. The correlation function values are shown in Table 1.

It can be seen from Table 1 that the maximum correlation coefficient (cc) is 0.535 , indicating that the original signal contained in IMF6 has the most principal components, so this information component is mainly maintained, and other components have larger noise components. Wavelet threshold processing is performed 
Table 1. Correlation coefficient between intrinsic mode and original signal.

\begin{tabular}{lllll}
\hline Modal layered & IMF1 & IMF2 & IMF3 & IMF4 \\
\hline cc & 0.501 & 0.296 & 0.303 & 0.399 \\
Modal layered & IMF5 & IMF6 & IMF7 & IMF8 \\
cc & 0.128 & 0.535 & 0.041 & 0.021 \\
Modal layered & IMF9 & IMF10 & IMF11 & $/$ \\
cc & 0.015 & 0.024 & 0.001 & $/$ \\
\hline
\end{tabular}
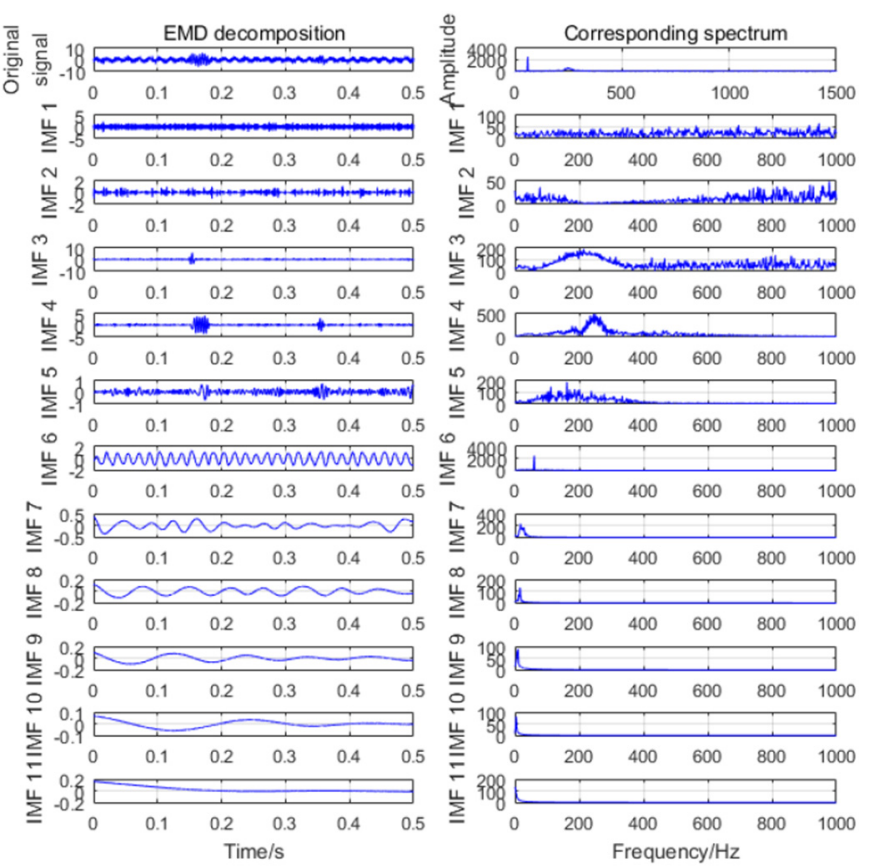

Fig. 4. Time-domain and frequency-domain diagrams of intrinsic mode components after wavelet processing.

on the intrinsic mode components of noise or interference with smaller correlation coefficients. The wavelet basis selected "db4" wavelet, the wavelet order is 5 layers, and the mode components of the output signal after wavelet threshold processing are shown in Figure 4. Further, the signal is reconstructed to obtain a signal with high performance after filtering. The reconstructed signal is shown in Figure 5.

Finally, we analyse the performance of this algorithm under the condition of varying signal-to-noise ratio. It can be seen from Table 2 and Figure 6 that as the input signalto-noise ratio increases, the output signal-to-noise ratio increases; at the same time the root mean square error decreases and the signal-to-noise ratio gain increases. It shows that the algorithm proposed in this paper is feasible to reduce noise with the presence of high-frequency intermittent signal interference and white noise interference.

The method proposed in this paper is compared with EMD method and the wavelet threshold denoising method. The performance comparison results are shown in Table 3. The combined method in this paper has better denoising
Table 2. Performance comparison under different input signal-to-noise ratios.

\begin{tabular}{rlll}
\hline SNR0 & SNR & RMSE & G \\
\hline-15 & -4.187 & 1.145 & 10.813 \\
-10 & -0.206 & 0.724 & 9.794 \\
-5 & 2.953 & 0.503 & 7.953 \\
0 & 7.733 & 0.41 & 7.733 \\
5 & 11.613 & 0.33 & 6.613 \\
10 & 18.494 & 0.31 & 8.494 \\
15 & 26.612 & 0.26 & 11.612 \\
\hline
\end{tabular}

Table 3. Performance comparison of different methods.

\begin{tabular}{llll}
\hline Algorithm & Proposed algorithm & EMD & WTD \\
\hline SNR & 7.733 & 1.616 & 1.088 \\
RMSE & 0.410 & 1.347 & 1.393 \\
\hline
\end{tabular}

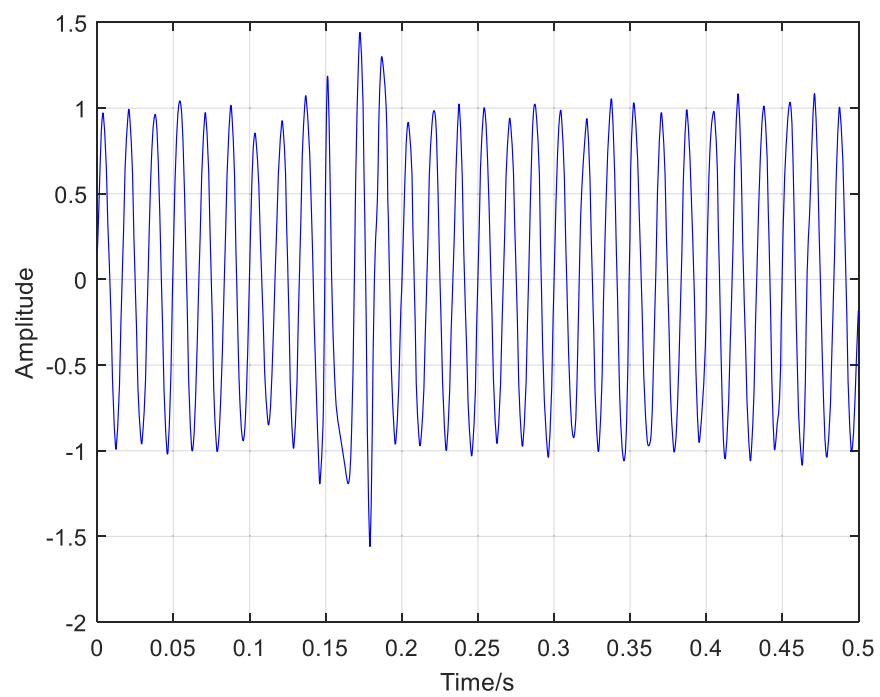

Fig. 5. Effective signal.

performance and small root mean square error. Therefore, the method proposed in this paper is effective for the extraction of intermittent signals.

The actual underwater acoustic signal measured by B\&K8104 hydrophone is used for noise reduction. The actual target is $315 \mathrm{~Hz}$ and $500 \mathrm{~Hz}$, and the others are interference and noise components. The method is applied to the test results of the actual field test. Figure 7 is the original test signal, and Table 4 is the correlation coefficients of each mode.

It can be seen that there are many signal components in IMF2 and IMF3. Then the algorithm proposed in this paper is used for signal processing, and the results are shown in Figure 8. The actual processing results show that the method can correctly process the actual signal, which has the significance of practical engineering application. 


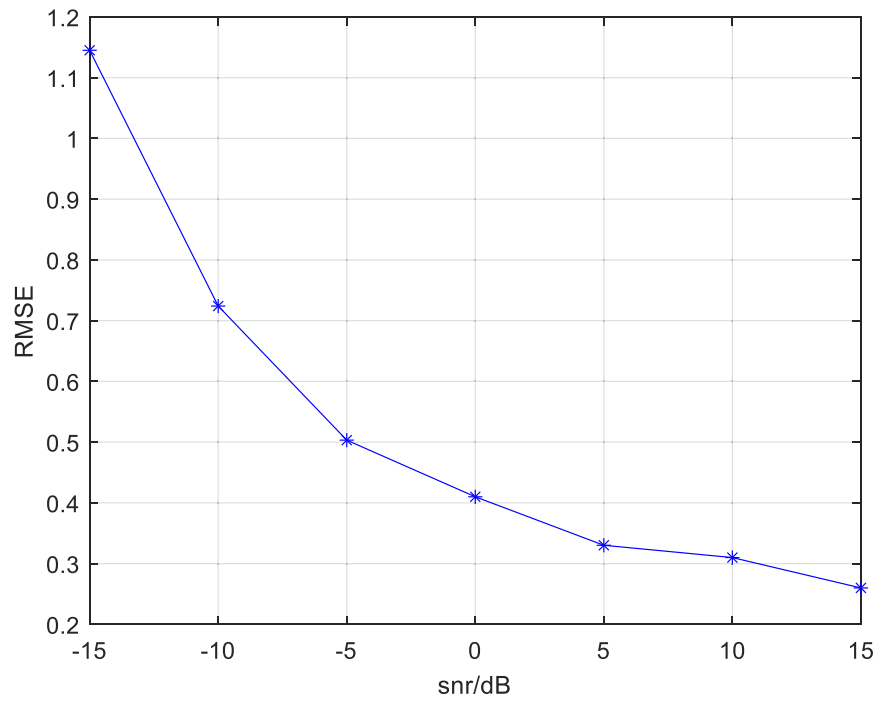

Fig. 6. Root mean square error curve under different signal-tonoise ratio.
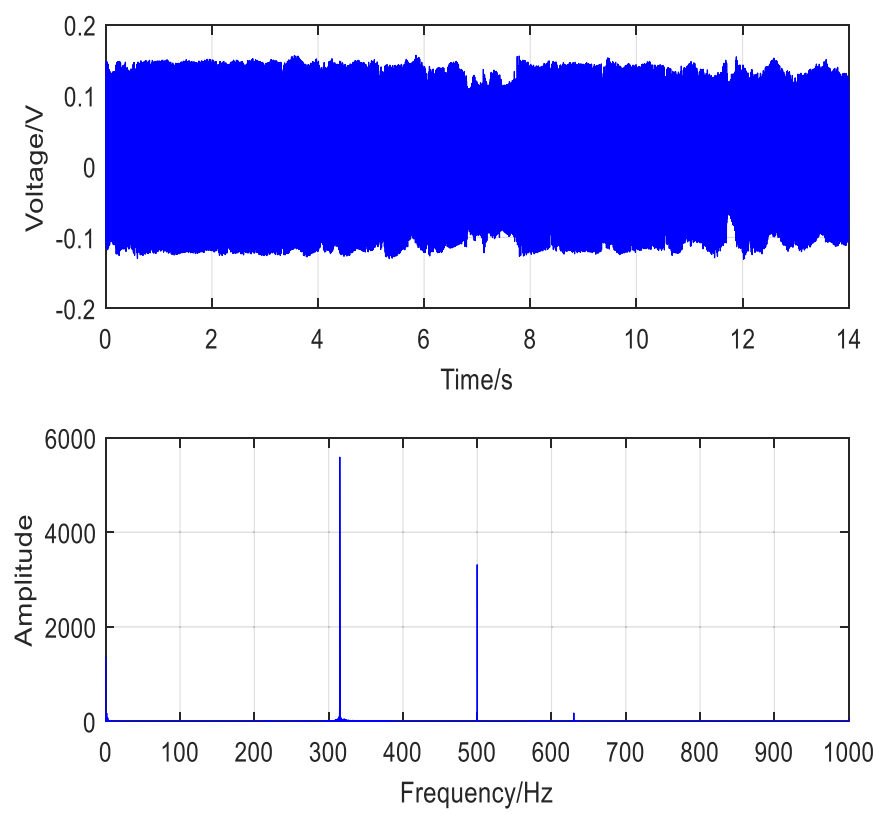

Fig. 7. Original test signal.

Table 4. Correlation coefficient between intrinsic mode and original signal.

\begin{tabular}{lllll}
\hline Modal layered & IMF1 & IMF2 & IMF3 & IMF4 \\
\hline cc & 0.216 & 0.722 & 0.617 & 0.107 \\
Modal layered & IMF5 & IMF6 & IMF7 & \\
cc & 0.016 & 0.003 & 0.0006 & \\
\hline
\end{tabular}

\section{Conclusion}

In this paper, the combined algorithm of EMD decomposition and wavelet transform is used to reduce the noise in
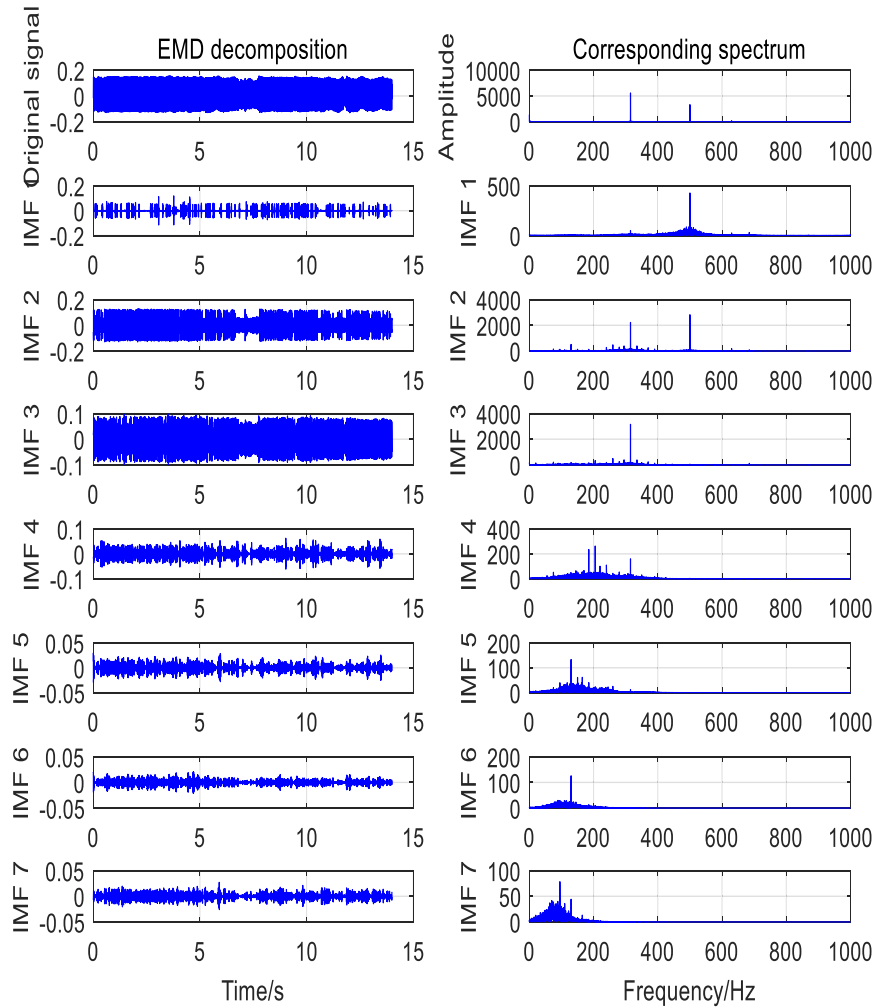

Fig. 8. Time-domain and frequency-domain diagrams of intrinsic mode components after wavelet processing.

the received signal in the underwater acoustic noise environment. Firstly, the calibration of baseline drift is performed, and then the EMD is decomposed into different intrinsic mode functions. The wavelet threshold processing is performed according to correlation coefficient of each mode component and the original signal. Finally, the signal is reconstructed. In this way, the useful part of the signal is effectively retained. The feasibility of the method proposed in this paper is verified by simulation. High-frequency intermittent signals and noise signals can be well extracted and removed, which provides the guarantee for the subsequent processing of underwater acoustic orientation algorithm. However, due to the time-varying and complexity of the underwater acoustic environment during practical testing, the performance of the algorithm in this paper under complex hydrological conditions requires further verification and improvement.

This work was supported by The Science and Technology Innovation Project of Shanxi Higher Education Institution (2020L0718), The Teaching Reform Project in Higher School in Shanxi (J2020383), Cooperative education project of industry university cooperation (201901157012).

\section{References}

1. L. Qihu, Sonar technology in entering 21 century, Signal Process. 28, 1-11 (2012) 
2. L. Qihu, New advances of underwater acoustic signal processing, Appl. Acoust. 31, 2-9 (2012)

3. H. Zuoyong, Review of some aspects of underwater noise including its control techniques and its prospect, Appl. Acoust. 21, 26-34 (2002)

4. A. Nehorai, E. Paldi, Acoustic Vector-Sensor Array Processing, IEEE Trans Signal Processing. 42, 2481-2491 (1994)

5. X. Pan, X. Xi, Structure design and performance research of a new co-vibration vector hydrophone, Ship Sci. Technol. 39, 148-168 (2017)

6. C. Yang, C. Aichen, S. Xiang, D. Bin, Research progress of fiber-optical sensors for ocean sensing and detection, J. Ocean Technol. 36, 1-9 (2017)

7. L. Bin, W. Peng, B. Yanping, A Combination algorithm for MEMS hydrophone signal and noise separation, Chin. J. Sens. Actuat. 27, 1477-1481 (2014)

8. N.E. Huang, Z. Shen, S.R. Long et al., The empirical mode decomposition and the Hilbert spectrum for nonlinear and non-stationary time series analysis, Proc. Roy. Soc. A: Math. Phys. Eng. Sci. (1998)
9. S. Zhenzhen, Z. Guojun, H. Jianjun, D. Junwen, Y. Shenghui, Using wavelet transform for signal processing of MEMS vector hydrophone, Ship Sci. Technol. 40, 130-134 (2018)

10. W. Shuanzhong, Z. Yutian, Comparative Simulation Experiment and Analysis of Improved Wavelet Threshold Denoising Method [J], Noise Vibr. Control 32, (2012)

11. N.E. Huang, A New Method for Non-linear and Nonstationary Time Series Analysis: The Hilbert Spectral Analysis [J], Proceedings of SPIE - The International Society for Optical Engineering. 4056, 197-209 (2000)

12. R.J. Urick, Principle of Underwater Sound (Harbin Institute of Marine Engineering Press, Harbin, 1990)

13. P. Yuhua, Wavelet Transform and Engineering Application [M], Beijing: Science Press, (1999)

14. Donoho. De-noising by soft-thresholding [J], IEEE Transactions on Information Theory. 41, 613-627 (1995)

15. X. Mingyuan, L. Zengli et al., Application of MATLAB Simulation in Signal Processing [M], Xi'an: University of Electronic Science and Technology of China Press 313-316 (2007)

Cite this article as: Junbing Shi, Yingmin Wang, Xiaoyong Zhang, Libo Yang, Extraction method of weak underwater acoustic signal based on the combination of wavelet transform and empirical mode decomposition, Int. J. Metrol. Qual. Eng. 12, 7 (2021) 